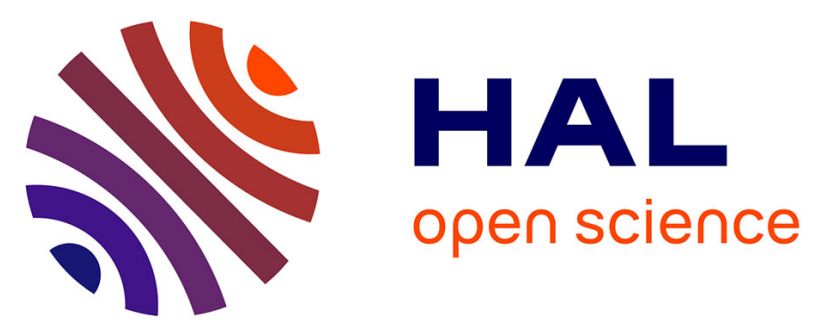

\title{
LES SERVICES AUX RETRAITÉS EN MILIEU RURAL : UN GISEMENT D'EMPLOIS ENTRE LOGIQUE PROVIDENTIELLE ET LOGIQUE CONCURRENTIELLE
}

Hélène Blasquiet-Revol, Clovis Sabau, Pauline Le Huidoux, Jean-François

Mamdy

\section{To cite this version:}

Hélène Blasquiet-Revol, Clovis Sabau, Pauline Le Huidoux, Jean-François Mamdy. LES SERVICES AUX RETRAITÉS EN MILIEU RURAL : UN GISEMENT D'EMPLOIS ENTRE LOGIQUE PROVIDENTIELLE ET LOGIQUE CONCURRENTIELLE. Pour, revue du Groupe Ruralités, Éducation et Politiques, 2011, 208, pp.165-171. hal-02122279

\section{HAL Id: hal-02122279 \\ https://hal.science/hal-02122279}

Submitted on 7 May 2019

HAL is a multi-disciplinary open access archive for the deposit and dissemination of scientific research documents, whether they are published or not. The documents may come from teaching and research institutions in France or abroad, or from public or private research centers.
L'archive ouverte pluridisciplinaire HAL, est destinée au dépôt et à la diffusion de documents scientifiques de niveau recherche, publiés ou non, émanant des établissements d'enseignement et de recherche français ou étrangers, des laboratoires publics ou privés. 
CHERCHER, REPÉRER, AVANCER

\section{LES SERVICES AUX RETRAITÉS EN MILIEU RURAL : UN GISEMENT D'EMPLOIS ENTRE LOGIQUE PROVIDENTIELLE ET LOGIQUE CONCURRENTIELLE}

Hélène Blasquiet-Revol, Clovis Sabau, Pauline le Huidoux et Jean-François Mamdy

GREP | « Pour »

2011/1 N²08 | pages 165 à 171

ISSN 0245-9442

Article disponible en ligne à l'adresse :

https://www.cairn.info/revue-pour-2011-1-page-165.htm

Distribution électronique Cairn.info pour GREP.

(C) GREP. Tous droits réservés pour tous pays.

La reproduction ou représentation de cet article, notamment par photocopie, n'est autorisée que dans les limites des conditions générales d'utilisation du site ou, le cas échéant, des conditions générales de la licence souscrite par votre établissement. Toute autre reproduction ou représentation, en tout ou partie, sous quelque forme et de quelque manière que ce soit, est interdite sauf accord préalable et écrit de l'éditeur, en dehors des cas prévus par la législation en vigueur en France. Il est précisé que son stockage dans une base de données est également interdit. 


\section{Les services aux retraités en milieu rural : un gisement d'emplois entre logique providentielle et logique concurrentielle}

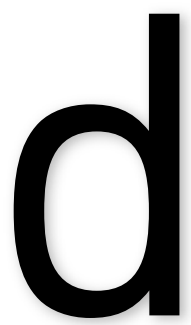

epuis deux décennies, l'attractivité du monde rural ne se dément pas. Les retraités représentent le quart des migrants s'installant dans l'espace rural ${ }^{1}$. Participant au vieillissement des campagnes, ces retraités induisent une demande de services qualifiés de services à la personne. Entre 1994 et 2004, le nombre de salariés du secteur des services à la personne (parmi lesquels figurent les services d'aide aux personnes âgées) a presque doublé ${ }^{2}$. Le dynamisme du secteur n'est pas prêt de faiblir avec la montée en puissance de l'aide à domicile pour des personnes âgées de plus en plus nombreuses 11 à 2 millions de personnes de plus de 85 ans dans le monde rural d'ici 2015).

Contact : VetAgro Sup, UMR Métafort, 89 Avenue de l'Europe, BP35, 63370 Lempdes h.blasquietavetagro-sup.fr ; c.sabauQvetagro-sup.fr ; pauline.le-huidouxQlive.fr ; jf.mamdy@ivetagro-sup.fr $1 \mathrm{~J}$.-F. Poncet et C. Belot, Rapport d'information sur le nouvel espace rural français, $n^{\circ} 468$, Sénat, 2008. 
Cependant, le développement de ces services s'opère difficilement dans un univers complexe encore largement dominé par le secteur public. Le potentiel de création d'activités de services est pourtant considérable, pour le secteur privé notamment. Cet article se propose de dresser un panorama des activités de services à la personne à destination des retraités résidant en milieu rural. Sur la base de l'analyse de deux territoires d'Auvergne lle Pays des Combrailles et la Communauté de communes de Billom-SaintDier), nous avons pu observer deux logiques à l'œuvre, l'une providentielle, l'autre concurrentielle, selon le statut, public ou privé, du créateur de l'activité de services.

\section{Les structures publiques, les prestataires et financeurs traditionnels de services aux retraités}

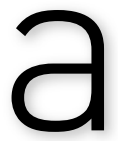
vec la création en 2005 de l'ANSP ${ }^{3}$, l'État consacre et encadre le secteur des services à la personne (SAP) ${ }^{4}$. Outre le rôle de régulateur lagrémentation de l'activité, encadrement du Cesu ${ }^{5}$...), de coordinateur (ARS ${ }^{6}$, Coderpa ${ }^{7}, \mathrm{Clic}^{8}$ ) et de planificateur (Plan stratégique régional de santé, Schéma départemental gérontologique, Schéma intercommunal des services publics...) de l'État et des collectivités locales, les structures publiques ont compétence pour la prestation ou le subventionnement de services à destination de la population retraitée.

La mise en place d'une politique de SAP s'organise la plupart du temps à l'échelle communale (CCAS ${ }^{9}$ ) ou intercommunale (Cias ${ }^{10}$, Sivos ${ }^{11}$ ) et relève alors d'une prise de compétence facultative. Leur émergence est donc le fruit de volontés politiques et traduit un souci de répondre à des besoins ressentis localement et identifiés par les acteurs locaux (élus, personnels médico-sociaux, proches, voisins...) ou extérieurs.

\footnotetext{
3 Agence nationale des services à la personne ; créée par la loi 2005-841 du 26 juillet 2005 relative au développement des services à la personne et portant diverses mesures en faveur de la cohésion sociale. 4 Selon la loi n $2005-841$ du 26 juillet 2005, sont regroupées parmi les prestataires de SAP « les associations et les entreprises dont l'activité porte sur la garde des enfants ou l'assistance aux personnes âgées, aux personnes handicapées ou aux autres personnes qui ont besoin d'une aide personnelle à leur domicile ou d'une aide à la mobilité dans l'environnement de proximité favorisant leur maintien à domicile et les centres communaux et intercommunaux d'action sociale au titre de leur activité de garde d'enfants de moins de trois ans à domicile ».

5 Chèque emploi-service universel.

6 Agence régionale de santé.

7 Comité départemental des retraités et des personnes âgées.

8 Centres locaux d'information et de coordination gérontologique.

9 Centre communal d'action sociale.

10 Centre intercommunal d'action sociale.

11 Syndicat intercommunal à vocation sociale.

12 Pour exemple, l'ADMR (Aide à domicile en milieu rural) a été fondée en 1945 (sous le nom de Aide familiale rurale à l'époque).
} 
L'apparition de ces services procède d'une démarche généralement ancienne et progressive sur les territoires. À Billom, le Sivos est né en 1984 de la volonté des élus de développer un service d'aide à domicile en réponse à un besoin ressenti sur le territoire, de structurer l'offre d'aide ménagère et d'assurer une gestion partagée du coût de cette compétence sociale.

Ces services peuvent être soit internalisés dans la structure - en régie directe -, soit délégués à un mandataire qui, de par une expérience reconnue ou une antériorité sur le territoire, est à même d'assurer le service en toute confiance pour le mandant ll'organisme public) et le bénéficiaire (le retraité). Dans ce deuxième cas de figure, la plupart du temps, le mandant est en lien avec un réseau associatif très développé sur son territoire. Ancrées historiquement et territorialement, ces associations ont pour objet la lutte contre les exclusions, l'assistance à domicile et les services à la personne d'une manière générale. Leur installation remonte souvent à plus de 50 ans ${ }^{12}$. Sur le territoire des Combrailles, on recense deux associations de ce type : l'association Aide au confort et au maintien à domicile, et l'association Aide ménagère basées à Saint-Éloy-lesMines. Il est très difficile de dessiner les contours de cette forme d'organisation, tant les aires d'actions peuvent être étendues et se recouper. Elles prennent souvent naissance de la volonté d'individus d'agir au service de leurs concitoyens dépendants.

Par ailleurs, de nombreux dispositifs de subventionnement de services existent ; collectivités et organismes publics offrent au retraité un large éventail d'aides selon ses moyens laide sociale aux personnes âgées, attribuée sous des critères d'âge et de revenu), son état de santé (allocation personnalisée d'autonomie, attribuée selon le degré de dépendance), ses besoins (défiscalisation plafonnée de recours à des prestations de services rémunérées en Cesu), ses conditions de logement... Ainsi, l'exemple de la Caisse des mines dans les Combrailles est caractéristique : créée avec le régime minier en 1946, elle dispose de deux antennes à Messeix et Saint-Éloy-lesMines qui comptaient chacune un millier de mineurs. Les mineurs et anciens mineurs possèdent des droits propres, notamment en matière de protection sociale et sanitaire (soins infirmiers et médicaux gratuits et sans avance de frais, aides aux logements et au chauffage, services d'aide à domicile, transport en ambulances, funérailles...) qui s'ajoutent à l'ensemble des services déjà présents sur le territoire.

Du fait de besoins croissants en services d'aide à la personne plus ou moins diversifiés (tels les menus travaux de jardinage, de rangement, de plomberie ou d'électricité... moins directement liés à des besoins médico-sociaux), le marché des services à la personne s'étend et s'étoffe. Nombre de ces services peuvent être réalisés par des proches (famille, amis, voisins), mais, dans de nombreux territoires ruraux, telles les Combrailles, les enfants ont migré vers les pôles urbains éloignés, et le voisinage se fait rare (nombreuses résidences secondaires) et peu disponible ${ }^{13}$. Sachant que ces services ne dépendent pas de compétences 
attribuées aux collectivités territoriales, qui n'ont donc pas obligation à les assurer, et qu'ils représentent un coût élevé ${ }^{14}$, un besoin et un potentiel d'activités apparaît ainsi dans les espaces ruraux, auxquels le secteur public n'est pas à même de répondre. Dès lors, des opportunités de marché apparaissent dont le secteur privé pourrait se saisir.

\section{Le secteur privé et la logique de marché, des pistes émergentes mais délicates}

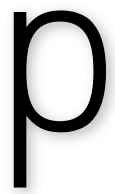

ris dans un sens large, les services à la personne privés rassemblent l'ensemble des prestations d'un individu au bénéfice d'un autre ${ }^{15}$. Du fait de l'éparpillement des prestataires et des bénéficiaires en milieu rural, le marché de l’offre privée est peu organisé. L'apparition des Cesu en 2006 régularise et simplifie la marchandisation de ces services. Le prestataire de SAP dispose désormais de la possibilité d'être agréé à titre simple ${ }^{16}$ ou agréé qualité ${ }^{17}$. L'agrémentation simple n'est pas obligatoire mais permet d'obtenir des avantages fiscaux. Cependant, en ce qui concerne les services pour les personnes âgées lassistance personnelle à domicile, aide à la mobilité et au transport, accompagnement dans les promenades et les tâches de la vie courante...), l'agrément qualité est obligatoire (pour un prestataire privé comme pour une association) ${ }^{18}$. Les entreprises de SAP sont émergentes en milieu rural, en contraste avec les territoires urbains ou périurbains. Sur 64 entreprises agréées recensées en 2009 dans l'en-

13 Contrairement à la Communauté de communes de Billom-Saint-Dier, où la proximité de Clermont-Ferrand et la densité de population permettent davantage aux retraités de compter sur la solidarité des proches.

14 En 2009, l'APA représente $7.5 \%$ du budget total des départements (pour 1136000 bénéficiaires) et les aides ciblées Personnes âgées, $3.9 \%$ (Chiffres DREES et DGCL 2010).

15 Ces services peuvent être proposés bénévolement ou rémunérés; officiellement lgré-à-gré, emploi direct, auto-entrepreneuriat) ou non (travail au noir, emploi dissimulé).

16 L'agrément simple est une autorisation à exercer certaines activités de SAP. Il est défini dans le Code du travail à l'article L-7232-I. Il donne droit à des avantages fiscaux et sociaux pour le prestataire du service.

17 L'agrément qualité est une autorisation obligatoire pour certaines activités de SAP à destination d'un public dit fragile.

18 art. L7231-1 du Code du travail.

19 La demande d'agrément qualité d'une entreprise de SAP varie selon les départements, et requiert la fourniture à l'autorité délibérante de nombreuses pièces justificatives incluant les éléments permettant d'apprécier le niveau de qualité des services mis en œuvre, des modèle de document d'information des clients et des usagers en matière fiscale, tarifaire, publicitaire, d'accueil, un document financier comprenant un budget prévisionnel, une étude de marché précise... (cf. art. R7232-1 et suivants du Code du travail).

20 Alors que les associations bénéficient de leurs propres réseaux, de leur antériorité et de leur expertise pour les appuyer dans le montage de leur activité.

21 Le Conseil d'analyse stratégique et la Dares publiaient en 2007 une étude sur l'évolution des métiers à horizon 2015. La population âgée, même si elle est uniquement considérée sous une thématique médicosociale dans l'étude, est supposée pourvoir 840000 emplois en France en 2015, soit une croissance annuelle du secteur de $2,7 \%$. 
semble du département du Puy-de-Dôme, 53 entreprises ont leur siège social situé en zone urbaine et périurbaine ; une seule de ces entreprises a été identifiée dans la Communauté de communes de Billom-Saint-Dier, et deux dans les Combrailles. Ces entreprises sont la plupart du temps dédiées à l'aide aux travaux de la vie courante (petits travaux de jardinage, prestations de petit bricolage, livraison de courses à domicile...), dessinant les contours du nouveau métier d'homme toutes mains, que certains prestataires revendiquent. Néanmoins, la complexité de mise en œuvre, due notamment aux exigences réglementaires inhérentes à la démarche d'agrémentation ${ }^{19}$, constitue un des freins majeurs identifiés pour l'installation d'individuels privés ${ }^{20}$, auxquels seule l'ANSP est à même d'apporter un soutien spécifique.

Les entreprises de SAP sont un secteur de l'entre-deux, elles réalisent des prestations qui pourraient être réalisées par des professionnels spécifiques mais dont l'ampleur réduite les dissuade de se déplacer, sinon à des coûts démesurés. Par exemple, une tonte de pelouse ne saurait être réalisée par un paysagiste, ou du petit bricolage (montage de meuble, changer une ampoule ou un joint de tuyauterie) par un ébéniste/électricien/plombier, etc. Ainsi, même si la cohabitation avec les artisans locaux est souvent délicate au commencement de l'activité de service (du fait du flou qui règne dans la définition des contours des prestations réalisables, et d'une crainte de concurrence déloyale), l'usage montre une complémentarité et une place pour chacun sur les territoires.

L'émergence du projet de SAP est souvent motivée par des attentes liées à l'indépendance et au bien-être personnel de l'entrepreneur, plutôt qu'à la volonté de satisfaire des besoins locaux. De fait, les prestataires privés intègrent progressivement les retraités et leurs besoins dans leur fonctionnement. Le service à la personne privé, quand il n'est pas conçu exclusivement à destination des retraités dans un premier temps, le devient ainsi par différentes adaptations (mise en place de livraisons à domicile par exemple) lorsque cette clientèle est très présente sur le territoire d'activité du prestataire (ce qui est le cas en milieu rural). L'appréhension des besoins se fait alors de manière spontanée, au sein de réseaux informels.

\section{Services à la personne publics et privés : entre concurrence et complémentarité}

es services à la personne publics et privés destinés aux retraités connaissent une forte croissance depuis

ces dernières années ${ }^{21}$. Toutefois, le secteur

public semble pour l'heure beaucoup plus structuré que ne l'est le 
secteur privé. En effet, les entreprises du secteur privé reconnaissent manquer de repères, de reconnaissance et de coordination entre elles. Les réseaux ne sont pas encore formalisés et on peut noter l'absence d'instances représentatives ${ }^{22}$, ou corporatistes, du secteur. Cependant, l'ANSP semble être vouée à jouer ce rôle, même si elle manque encore de moyens au regard de l'enjeu 23.

Nombre de praticiens des territoires interrogés ont tendance à percevoir les entreprises de SAP comme une mise en cause du service public, étant de fait amenées à proposer la même gamme de services que le public. De plus, lier le social et le profit économique est encore une idée peu répandue et peu acceptée, ce qui justifie la place prégnante tenue par le secteur associatif dans le domaine. Il est en outre reproché aux prestataires privés de manquer de professionnalisme, de rigueur et de pérennité dans leurs projets. À l'inverse, ils reprochent au public un défaut de sensibilité, d'empathie et de relation de confiance avec le client, notamment dans le cas des services aux retraités où la dimension de proximité est essentielle.

Ces visions antagoniques sont avant tout le fruit d'une perception concurrentielle (tant économique qu'idéologique). Or, il peut exister une certaine entente, voire une complémentarité entre ces deux secteurs. Citons l'exemple de cette créatrice d'entreprise de services à la personne à Billom-Saint-Dier qui s'est rapprochée des structures d'aide à domicile publiques (Sivos et Relais d'assistantes maternelles) afin de pouvoir réaliser des prestations adaptées à une clientèle plus large, apportant par là même un complément de travail et de revenu aux salariés du public, souvent employés à temps partiel.

Modification des modes de vie et de consommation des seniors (vieillissement actif, silver economy, gérontechnologies, domotique...), croissance et diversification des besoins, ou bien isolement, dépendance et faible retraite agricole, et minière, légitiment le développement de nouveaux services à la personne publics et privés dans les espaces ruraux. Cependant pour des raisons de représentation, d'accessibilité, de lisibilité et de connaissance fine des besoins locaux, une coordination des professionnels s'impose, ce que le dispositif Clic 24 initie par ailleurs. Sous réserve de lever ces obstacles majeurs, le secteur des services à la personne pourrait être appelé à un bel essor, et devenir un levier majeur de création d'emplois en espace rural.

22 Au contraire du secteur de l'artisanat représenté par les chambres des métiers et de l'artisanat ou du secteur agricole avec les chambres d'agriculture par exemple.

23 Dans le Puy-de-Dôme, seule une personne est responsable de l'antenne locale de l'ANSP pour accompagner et informer les créateurs de SAP.

24 Centres locaux d'information et de coordination. 


\section{À lire}

B. Balzani, Les services à la personne, coll. «Études », La Documentation française, 2010.

C. Gallouj, L'économie des services à la personne, Wolters Kluwer France, 2008.

L. Grouas (dir.), C. Alisaïd-Guérin, C. Berbezier, A. Bertrand, M. Le Goff-Pronost et al., Les collectivités locales et la prise en charge de la dépendance des personnes âgées, Les classeurs de la lettre du cadre territorial $n^{\circ} 7$, Territorial Éditions, 2008.

J.-N. Lesellier, Les services à la personne, comment ça marche ?, $2^{\circ}$ édition, Wolters Kluwer France, 2009.

E. Mouhoud (dir.), Économie des services et développement des territoires, Coll. «Travaux », La Documentation française - Datar, 2010. 